\title{
Investigation of Materials Used in Synthesis for Silver Nanowires and Nanoparticles
}

\author{
Cheng-Tang Pan, ${ }^{1}$ Tsung-Lin Yang, ${ }^{1}$ Yi-Chian Chen, ${ }^{1}$ \\ Shin-Pon Ju, ${ }^{1}$ I-Chou Wu, ${ }^{1}$ Yu-Ru Lin, ${ }^{1,2}$ and Cherng-Yuh Su ${ }^{3 *}$ \\ ${ }^{1}$ Department of Mechanical and Electro-Mechanical Engineering, \\ National Sun Yat-Sen University, Kaohsiung, Taiwan, 70, Lienhi Rd., Kaohsiung 80424, Taiwan \\ ${ }^{2}$ Department of Engineering and System Science, National Tsing Hua University, Hsinchu, Taiwan \\ 101, Sec. 2, Kuang-Fu Rd., Hsinchu 30013, Taiwan \\ ${ }^{3}$ Department of Mechanical Engineering, National Taipei University of Technology, Taipei, Taiwan \\ 1, Sec. 3, Zhongxiao E. Rd., Taipei 10608, Taiwan
}

(Received August 31, 2015; accepted February 5, 2016)

Keywords: synthesis materials, silver nanowires, silver nanoparticles, ITO, TCF

Transparent conductive films (TCFs) with high transmittance in the visible light band and excellent conductivity are widely applied in the panel industry, and also in electronic paper and related optical industries. In addition to the prevalent TCF indium tin oxide (ITO), there are new materials suitable for use as TCFs, such as carbon nanotubes, graphene, and metal meshes. In this study, the polyol method was used to synthesize silver nanowires (AgNWs) and silver nanoparticles (AgNPs). Silver seeds and silver ions were combined to obtain AgNWs. Scanning electron microscopy was used to observe the morphology of the AgNWs. The influence of properties of different materials used in synthesis on the morphology of AgNWs was investigated. The properties include molecular weight (MW) of polyvinylpyrrolidone (PVP), the identity of the seed materials, and the source of the silver ions. The results show that a PVP MW of 1300000, AgCl as silver seeds, and $\mathrm{AgNO}_{3}$ as the source of silver ions yield uniform AgNWs. The average length of the wires was $1.7 \pm 0.3 \mu \mathrm{m}$, and the aspect ratio was $20.5 \pm 1.0$. The optical and electrical properties of thin films of AgNWs were analyzed. The transmittance of the thin films was $52 \%$, and the sheet resistance was $55 \mathrm{M} \Omega / \mathrm{sq}$.

\section{Introduction}

Recently, significant attention has been focused on applications of transparent electrodes due to the flourishing development of the high-tech industry and smart displays. ${ }^{(1-4)}$ Indium tin oxide (ITO) is the prevailing material used in transparent electrodes. ${ }^{(5,6)}$ However, disadvantages of $\operatorname{ITO}^{(7,8)}$ have encouraged the development of emerging alternatives to replace ITO. New materials have been developed for transparent conductive films (TCFs) in research in related areas such as metal meshes, ${ }^{(9)}$ graphene, ${ }^{(10)}$ carbon nanotubes, ${ }^{(11)}$ and silver nanowires (AgNWs). The AgNWs can be fabricated in many processes, and the experimental parameters can be easily controlled to obtain optimal AgNWs. The transmittance and conductivity of AgNWs can be the same as those of ITO. ${ }^{(12,13)}$ However, the flexibility of AgNWs is better than that of ITO, and the application of AgNWs to flexible substrates will create significant benefits.

${ }^{*}$ Corresponding author: e-mail: cysu@ntut.edu.tw 
Currently, two main processes involving the chemical and physical methods are used to synthesize silver nanoparticles (AgNPs) and AgNWs. The physical methods include a physical crushing method, a mechanical grinding method, and a laser ablation method. ${ }^{(14)}$ The chemical methods include a sol-gel method, a metal-phase synthesis method, ${ }^{(15)}$ and a chemical reduction method which reduces metal ions to atoms which self-assembled to from AgNPs. ${ }^{(16)}$ Several methods such as the template synthesis method, ${ }^{(17)}$ the seed generation method, ${ }^{(18)}$ a nanostructure self-assembly method, and a polyol reduction method ${ }^{(19-2)}$ have been used to synthesize AgNWs.

The polyol reduction method could be applied to synthesize AgNWs, and would offer low cost and a simple fabrication process. Moreover, the experimental parameters are easily controlled for the fabrication of nanosilver. In this study, the polyol reduction process was adopted, and the experimental parameters including the PVP molecular weight (MW), the seed material, and the source of silver ions were used to constitute the reactions. Scanning electron microscopy (SEM) was used to observe the morphology of AgNWs, and the four-point probe was used to measure the sheet resistance of the films. From the results, the optimal parameters for the synthesis of AgNWs were obtained.

\section{Materials and Methods}

\subsection{Starting materials for the synthesis of AgNWs and AgNPs}

The polyvinylpyrrolidone (PVP) was used as a surfactant, and $\mathrm{AgNO}_{3}(99.0 \%)$ was used to generate AgNPs. During the formulation of the solution, $\mathrm{AgCl}(99.999 \%)$ and $\mathrm{AgNO}_{3}$ were added to the solution as the silver seed and the source of silver ions, respectively. Ethylene glycol (EG, $99.5 \%$ ) was used as the reducing agent and solvent.

\subsection{Preparation of AgNWs and AgNPs}

The PVP was placed into a glass vessel and then the EG was added and mixed with the PVP. The solution was heated at constant temperature and stirred at a rate of $350 \mathrm{rpm}$ until the PVP was completely dissolved. Then, the $\mathrm{AgCl}$ as the silver seed was added to the solution. After continuous heating and stirring for $5 \mathrm{~min}, \mathrm{AgNO}_{3}$ was added to the solution.

\subsection{Experiments with conditions}

Figure 1 shows the flow chart for the fabrication and analysis of AgNPs. In the experiment, the different PVP MWs and different reaction times were adopted as control variables. Then, SEM was used to observe the morphology of AgNPs.

Figure 2 shows the flow chart for the fabrication and analysis of AgNWs. The variable factors include PVP MW, the seed material, and the source of silver ions. The influence of different properties of starting materials on the morphology of AgNWs was studied. Then the optical and electrical properties of $\mathrm{AgNW}$ thin film were analyzed. 


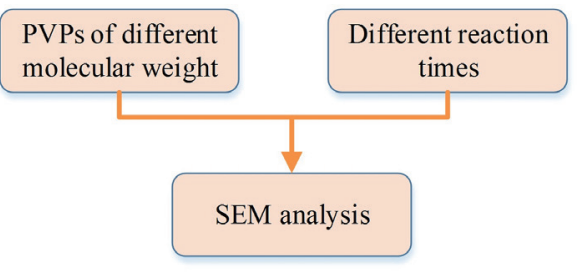

Fig. 1. (Color online) Flow chart for fabrication and analysis of AgNPs.

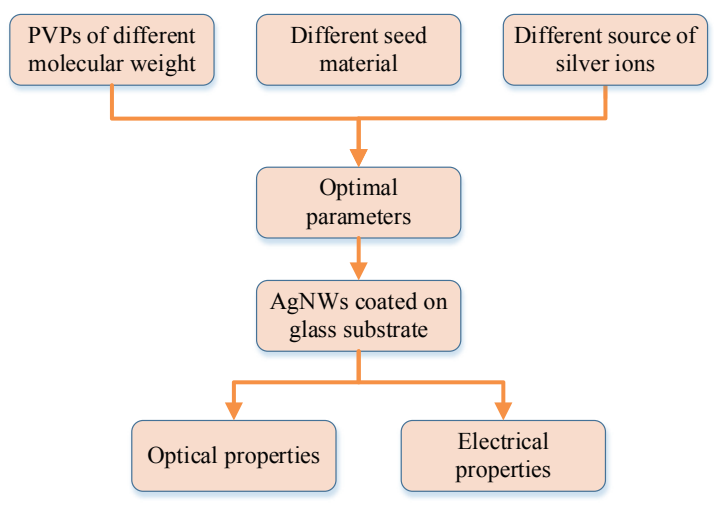

Fig. 2. (Color online) Flow chart for fabrication and analysis of AgNWs.

\section{Results and Discussion}

\subsection{Starting materials for the synthesis of AgNPs}

The influence of different MWs of PVP on the synthesized AgNPs was investigated. The following quantities $-5 \mathrm{ml} \mathrm{EG}, 0.025 \mathrm{~g} \mathrm{AgNO}_{3}, 0.1 \mathrm{~g} \mathrm{PVP}, 170{ }^{\circ} \mathrm{C}$ temperature, $7 \mathrm{~h}$ stirring time, and $350 \mathrm{rpm}$ stirring rate-were set as constant experimental parameters. PVP MWs of 1300000 and 58000 were adopted to synthesize the AgNPs and the morphologies of the AgNPs were observed. Figure 3 shows a comparison of the morphologies of AgNPs synthesized with different MWs of PVP. Figure 3(a) shows AgNPs $111 \pm 13 \mathrm{~nm}$ in diameter synthesized with PVP of MW 1300000. Figure 3(b) shows AgNPs $93 \pm 5 \mathrm{~nm}$ in diameter synthesized with PVP of MW 58000. Figure 4 shows the deviation in the diameter of AgNPs. The results show that the use of PVP of MW 58000 yields more uniform and smaller particle sizes.

Next, the influence of different reaction times on the AgNPs was investigated. The following quantities-20 ml EG, $0.1 \mathrm{~g} \mathrm{AgNO}_{3}, 0.4 \mathrm{~g} \mathrm{MW} 58000 \mathrm{PVP}, 170{ }^{\circ} \mathrm{C}$ temperature, and $350 \mathrm{rpm}$ stirring rate-were set as constant experimental parameters. The reaction time was set at (a) 5 , (b) 7, (c) 9, and (d) $11 \mathrm{~h}$. Figure 5 shows the morphologies of AgNPs synthesized at different reaction times. Figure 5(a) shows the AgNPs with a diameter of $43 \pm 4 \mathrm{~nm}$ synthesized at $5 \mathrm{~h}$. Figure 5(b) shows the AgNPs with a diameter of $80 \pm 4 \mathrm{~nm}$ synthesized at $7 \mathrm{~h}$. Figure 5(c) shows the AgNPs with a diameter of $85 \pm 3 \mathrm{~nm}$ synthesized at $9 \mathrm{~h}$. Figure 5(d) shows the AgNPs with a diameter of $89 \pm 4 \mathrm{~nm}$ synthesized at $11 \mathrm{~h}$. In addition, the deviation in the diameter of AgNPs is shown in Fig. 6. These results reveal that the diameter of AgNPs approaches a certain value. Because the source of silver is limited, the increased reaction time may cause the amount of silver dissolved in the solution to approach saturation. 


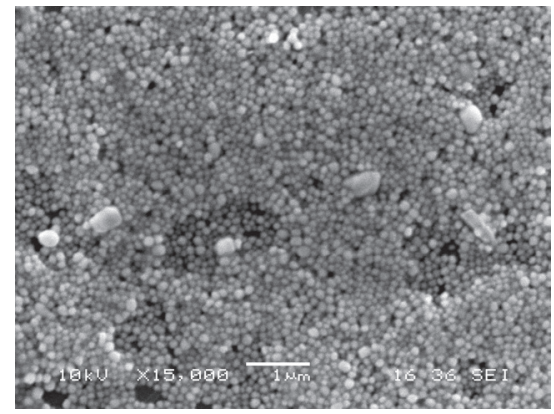

(a)

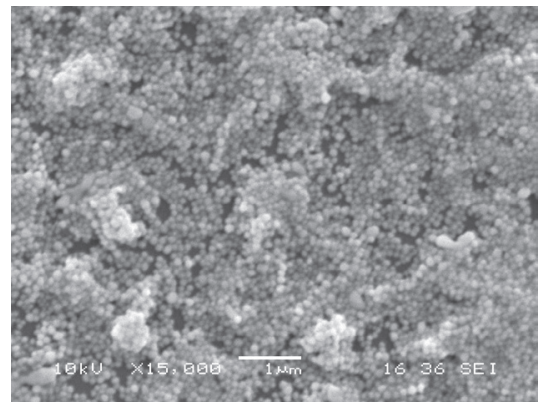

(b)

Fig. 3. The morphologies of AgNPs synthesized with different PVP MWs: (a) MW 1300000, (b) MW 58000.

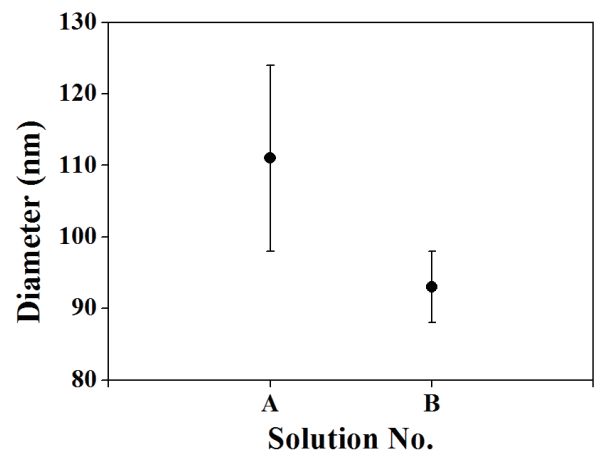

Fig. 4. Deviation in the diameter of AgNPs synthesized with different PVP MWs.

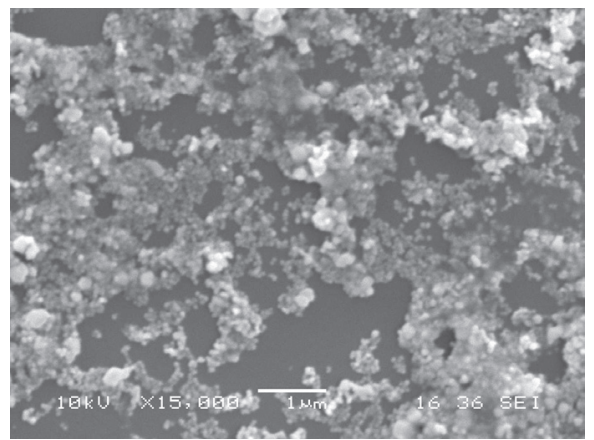

(a)

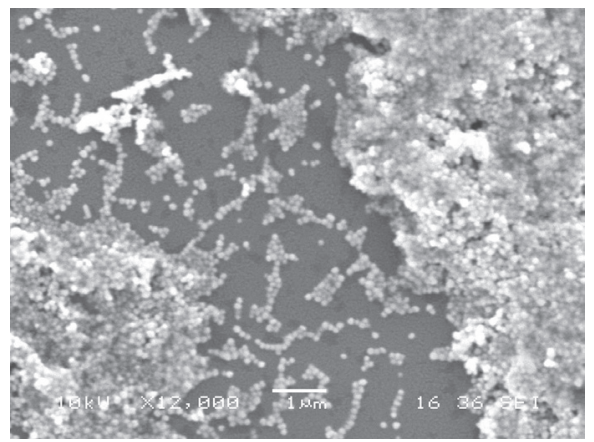

(c)

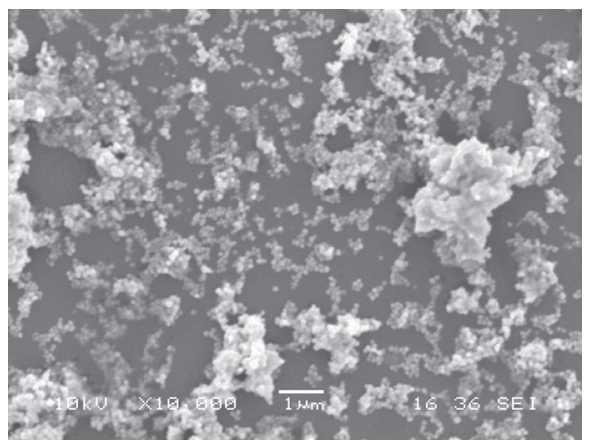

(b)

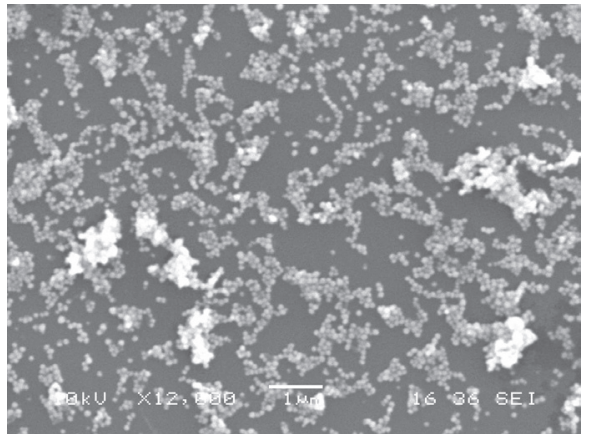

(d)

Fig. 5. The morphologies of AgNPs synthesized at different reaction times: (a) 5, (b) 7, (c) 9, and (d) $11 \mathrm{~h}$. 


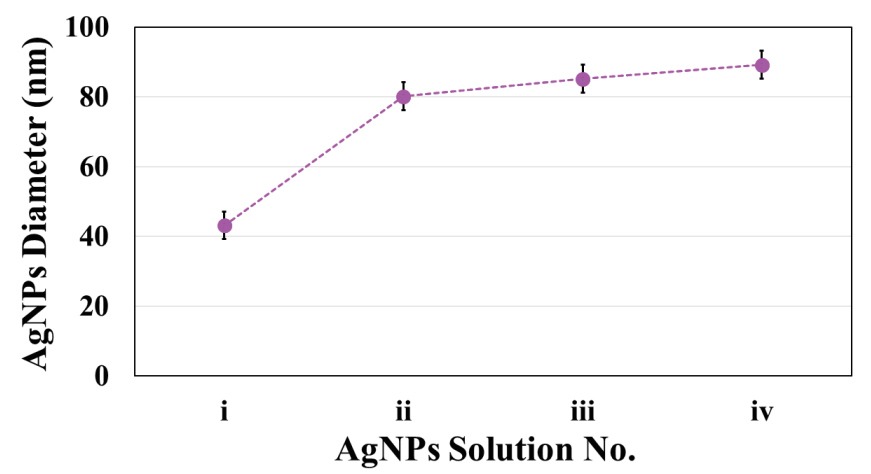

Fig. 6. (Color online) Deviation in the diameter of AgNPs synthesized at different reaction times.

\subsection{Starting materials for the synthesis of AgNWs}

The influence of different MWs of PVP on the AgNWs produced was investigated. The following quantities- $0.21 \mathrm{~g} \mathrm{PVP}, 0.22 \mathrm{~g} \mathrm{AgNO}_{3}, 0.05 \mathrm{~g} \mathrm{AgCl}, 20 \mathrm{ml} \mathrm{EG}, 170{ }^{\circ} \mathrm{C}$ temperature, $7 \mathrm{~h}$ stirring time, and $350 \mathrm{rpm}$ stirring rate-were set as constant experimental parameters. The MW 1300000 and MW 58000 PVPs were used to synthesize the AgNWs, and the morphologies of the AgNWs were observed. Figure 7 shows the comparison of morphologies of AgNWs synthesized with PVP of different MWs. Figure 7(a) shows that the AgNWs synthesized with PVP of MW 1300000 were longer and more uniform in diameter, whereas Fig. 7(b) shows the AgNWs synthesized with PVP of MW 58000 were shorter and irregular in diameter. The experimental results show that fewer AgNWs were produced and their diameter was irregular when the lower MW PVP was used. Presumably, because the lower MW PVP cannot form a high density of bonds between the Ag (100) plane and O in PVP, the PVP cannot completely attach to one side of the AgNWs and cause them to grow anisotropically. (22)

Next, the influence of different silver seed materials on the AgNWs was investigated. Four different seed materials- $\mathrm{AgBr}, \mathrm{NaCl}, \mathrm{KCl}$, and $\mathrm{AgCl}$ - were used to synthesize AgNWs. The following quantities - $0.21 \mathrm{~g} \mathrm{MW} 1300000 \mathrm{PVP}, 0.22 \mathrm{~g} \mathrm{AgNO}_{3}, 0.05 \mathrm{~g}$ silver seed, $20 \mathrm{ml} \mathrm{EG}, 170$ ${ }^{\circ} \mathrm{C}$ temperature, $7 \mathrm{~h}$ stirring time, and $350 \mathrm{rpm}$ stirring rate-were set as constant experimental parameters. Figure 8(a) shows the result of using $\mathrm{AgBr}$ as the silver seed. AgNPs with a smaller diameter are observed. Figure 8(b) shows the result of using $\mathrm{NaCl}$ as the silver seed. AgNPs with a larger but nonuniform diameter and few AgNWs are seen. Figure 8(c) shows the result of using $\mathrm{KCl}$ as the silver seed. AgNWs with a uniform diameter and AgNPs with nonuniform diameters are obtained. The AgNWs accounted for about $60 \%$ of the total amount of product. Figure 8(d) shows the result of using $\mathrm{AgCl}$ as the silver seed. Many AgNWs with uniform diameter are observed. These experimental results show that the adoption of $\mathrm{AgCl}$ as the silver seed results in a larger amount of more uniform AgNWs.

The influence of the source of silver ions on the AgNWs produced was explored. Silver nitrate and silver acetate were the two materials used as the sources of silver ions. The following quantities - $0.21 \mathrm{~g} \mathrm{MW} 1300000 \mathrm{PVP}, 0.22 \mathrm{~g}$ source of silver, $0.05 \mathrm{~g} \mathrm{AgCl}, 20 \mathrm{ml} \mathrm{EG}, 170{ }^{\circ} \mathrm{C}$ temperature, $7 \mathrm{~h}$ stirring time, and $350 \mathrm{rpm}$ stirring rate-were set as constant experimental 


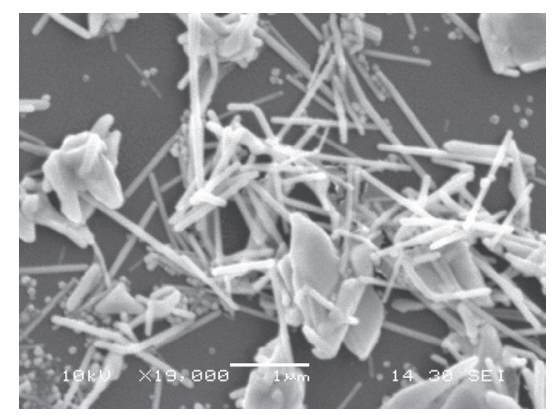

(a)

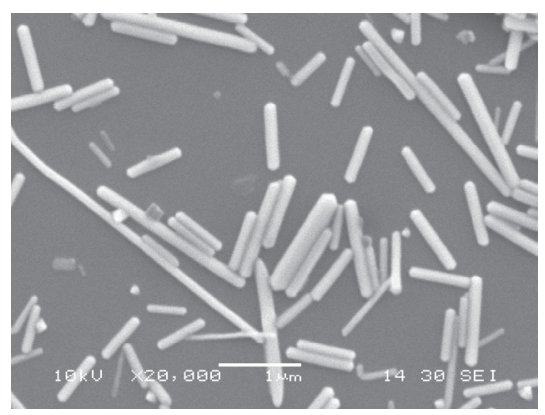

(b)

Fig. 7. The morphologies of AgNWs synthesized with PVP of (a) MW 1300000 and (b) MW 58000.

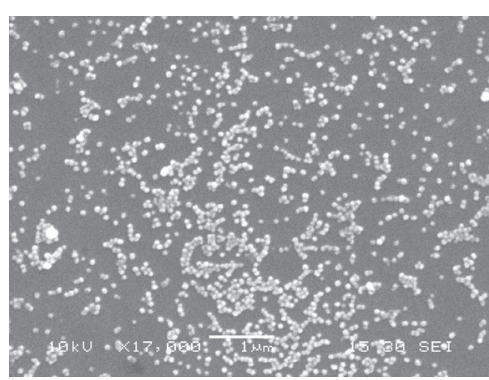

(a)

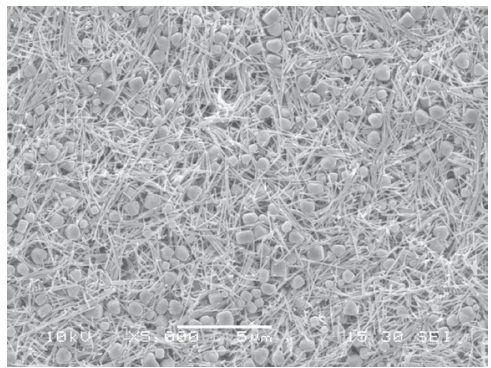

(c)

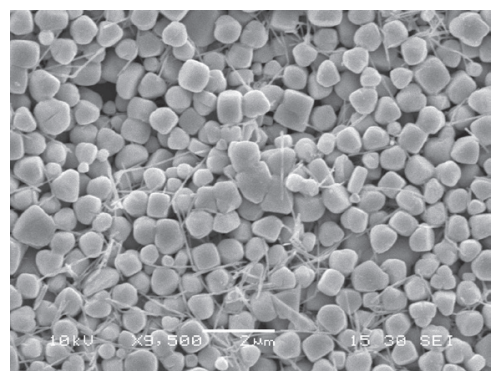

(b)

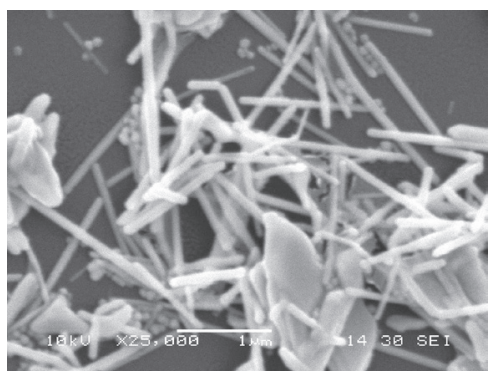

(d)

Fig. 8. The morphologies of AgNWs synthesized using different silver seeds: (a) AgBr, (b) NaCl, (c) KCl, and (d) $\mathrm{AgCl}$.

parameters. Figure 9(a) shows the result of using silver nitrate as the source of silver ions; uniform AgNWs were obtained. Figure 9(b) shows the result of using silver acetate as the source of silver ions; AgNPs were produced.

These results indicate that the adoption of MW 1300000 PVP, $\mathrm{AgCl}$ as the silver seed, and $\mathrm{AgNO}_{3}$ as the source of silver ions results in more uniform AgNWs. Their average length was $1.7 \pm 0.3$ $\mu \mathrm{m}$ and their aspect ratio was $20.5 \pm 1.0$.

Finally, the AgNWs were used to fabricate thin films with different transmittance. The transmittance of sample A was $18 \%$ for a thickness of $2918.0 \AA$. The transmittance of sample B was $45 \%$ for a thickness of $1661.7 \AA$. The transmittance of sample $\mathrm{C}$ was $52 \%$ for a thickness of $705.9 \AA$. The transmittance of sample D was $33 \%$ for a thickness of $2619.0 \AA$. The sheet resistances were measured using a four-point probe. Figure 10 shows the relation of transmittance 


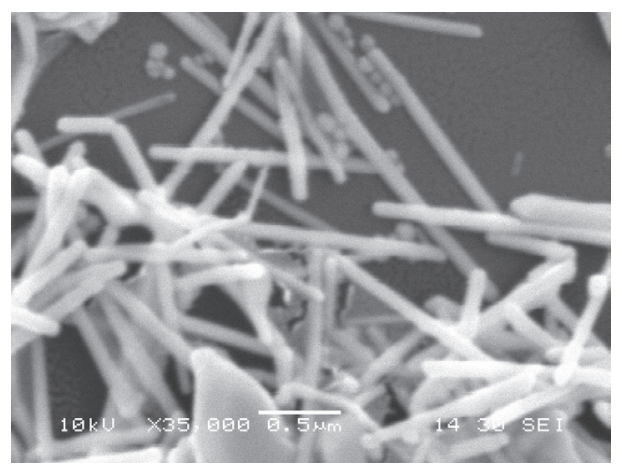

(a)

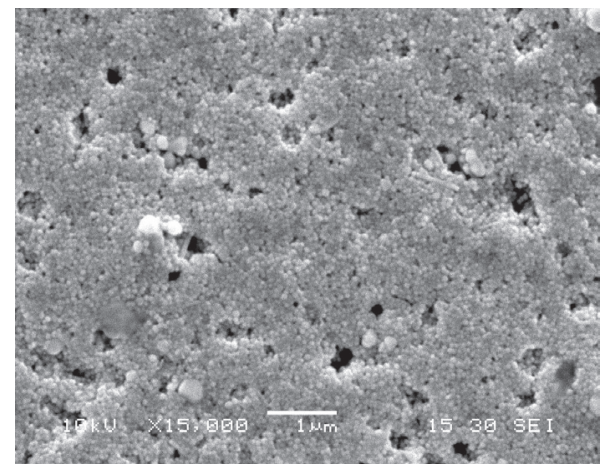

(b)

Fig. 9. The morphologies of AgNWs synthesized using different sources of silver ions: (a) silver nitrate and (b) silver acetate.

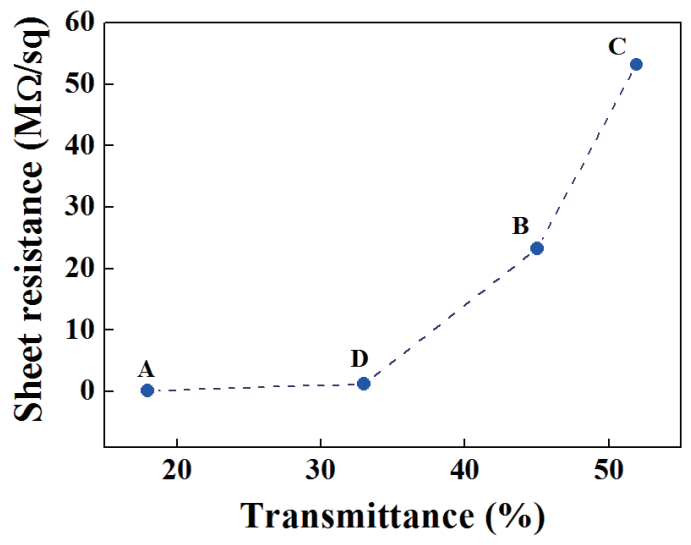

Fig. 10. (Color online) Relationship between transmittance and sheet resistance.

to sheet resistance. Sheet resistance and transmittance are inversely proportional. The optimal transmittance of an AgNW thin film was $52 \%$ and its sheet resistance was approximately $55 \mathrm{M} \Omega / \mathrm{sq}$.

\section{Conclusions}

The diameter and uniformity of AgNPs synthesized with PVP of MW 58000 were smaller and more even than those synthesized with PVP of MW 1300000. In addition, the diameter of AgNPs approached a certain limiting value. With the aspect ratio as a definable target for AgNWs, the PVP, silver seed, and source of silver ions were investigated. From the results, the use of the PVP of MW $1300000, \mathrm{AgCl}$ as the silver seed, and $\mathrm{AgNO}_{3}$ as the source of silver ions resulted in more uniform AgNWs.

\section{Acknowledgements}

The authors thank the Ministry of Science and Technology of Taiwan for their support of this research under Grants 103-2221-E-110-009-MY3 and the TCUS-ITRI for their support of this research under Grants 103 project support. 


\section{References}

1 J. Y. Zou, H. L. Yip, S. K. Hau, and A. K. Y. Jen: Appl. Phys. Lett. 96 (2010) 203301.

2 S. H. K. Park, C. S. Hwang, M. Ryu, S. Yang, C. Byun, and J. Shin: Adv. Mater. 21 (2009) 678

3 M. J. Alam and D. C. Cameron: Thin Solid Films 377 (2000) 455.

4 X. Liu, X. Cai, J. Mao, and C. Jin: Appl. Surf. Sci. 183 (2001) 103.

5 T. Minami: Thin Solid Films. 516 (2008) 5822.

6 J. Ouyang and Y. Yang: Adv. Mater. 18 (2006) 2141.

7 Y. Z. Lin, H. F. Dam, T. R. Andersen, E. Bundgaard, W. F. Fu, H. Z. Chen, F. C. Krebs, and X. W. Zhan: J Mater. Chem. C 1 (2013) 8007.

8 O. Inganas: Nat. Photon. 5 (2011) 201.

9 J. Y. Lee, S. T. Connor, Y. Cui, and P. Peumans: Nano Lett. 8 (2008) 689.

10 X. Wang, L. Zhi, and K. Mullen: Nano Lett. 8 (2008) 323

11 T. W. Ebbesen, H. J. Lezec, H. Hiura, J. W. Bennett, H. F. Ghaemi, and T. Thio: Nature 382 (1996) 54

12 H. H. Khaligh: Silver Nanowire Transparent Electrodes Fabrication, Characterization, and Device Integration (Applied Science, University of Waterloo, 2013).

13 A. R. Madaria, A. Kumar, F. N. Ishikawa, and C. Zhou: Nano Res. 3 (2010) 564.

14 G. B. Sergeev: Nanochemistry, 2nd ed. (Elsevier, 2006) p. 7.

15 C. Bréchignac, P. Houdy, and M. Lahmani (Eds.): Nanomaterials and Nanochemistry (Springer link, 2007) p. 395.

16 C. H. Yu, W. Oduro, K. Tam, and E. S. C. Tsang: Handbook of metal physics, ed. John, A. B (Elsevier, 2008) Chap. 10.

17 B. Sun, X. Jiang, S. Dai, and Z. Du: Mater. Lett. 63 (2009) 2570.

18 H. Yu, P. C. Gibbons, K. F. Kelton, and W. E. Buhro: J. Am. Chem. Soc. 123 (2001) 9198.

19 W. Yang, L. Qu, R. Zheng, Z. Liu, K. R. Ratinac, L. Shen, D. Yu, L. Yang, J. Barrow, and C. S. P. Ringer: Chem. Mater. 23 (2011) 2760.

20 L. K. Kurihara, G. M. Chow, and P. E. Schoen: Nanostructured Mater. 5 (1995) 607.

21 T. L. Yang, C. T. Pan, Y. C. Chen, L. W. Lin, I. C. Wu, K. H. Hung, Y. R. Lin, H. L. Huang, C. F. Liu, and S. W. Mao: Optical Mater. 39 (2015) 118.

22 K. H. Hung: The investigation of characteristics of electrospun Ag/polymer fibers and conductive films fabricated by Polyol reduction process, ed. (Applied Science, National Sun Yat-sen University, 2013). 